\title{
High Power Field
}

National Cancer Institute

\section{Source}

National Cancer Institute. High Power Field. NCI Thesaurus. Code C67274.

The area visible under the maximum magnification power of the objective being used in microscopy. 